\title{
Identifying survival-associated ceRNA clusters in cholangiocarcinoma
}

\author{
MING WAN $^{1 *}$, FU-MIN ZHANG ${ }^{1,2, *}$, ZHENG-LONG LI $^{1}$, PENG-CHENG KANG ${ }^{1}$, PING-MING JIANG ${ }^{1}$, \\ YI-MIN WANG ${ }^{1}$, ZHI-DONG WANG $^{1}$, XIANG-YU ZHONG ${ }^{1}$, CHUN-LONG LI ${ }^{1}$, \\ $\mathrm{HAO} \mathrm{WANG}^{1}$, SHI-YONG ZHAO ${ }^{1}$ and YUN-FU CUI ${ }^{1}$ \\ ${ }^{1}$ Department of Hepatopancreatobiliary Surgery, Second Affiliated Hospital of Harbin Medical University, Harbin, \\ Heilongjiang 150086; ${ }^{2}$ Department of General Surgery, Daqing Oilfield General Hospital, \\ Daqing, Heilongjiang 163000, P.R. China
}

Received February 6, 2016; Accepted March 17, 2016

DOI: 10.3892/or.2016.4934

\begin{abstract}
Competing endogenous RNAs (ceRNAs) represent a novel layer regulations of long non-coding RNAs (lncRNAs) and genes that play important roles in cancer pathogenesis by binding microRNAs (miRNAs). However, the competition mechanism of ceRNAs in cholangiocarcinoma (CHOL) is not fully understood. In this study, we constructed a dysregulated ceRNA competitive network (CCEN) to globally characterize the competing difference between CHOL and normal tissues. Then, we integrated affinity propagation and Kaplan-Meier (K-M) methods to identify functional clusters associated with survival. A total of 7 key ceRNA clusters were identified. Further functional annotation analyses found that Cluster23 and Cluster32 involved cell based functions, and the loss of ceRNA competitive relations in clusters may contribute to CHOL, by disturbing important biological processes, such as 'Pathway in cancer', MAPK and Neurotrophin signaling pathway. This study provides further insights into understanding the competitive mechanism of ceRNAs in CHOL.
\end{abstract}

\section{Introduction}

Cholangiocarcinoma (CHOL), which predominantly arises from the epithelial bile duct, is an aggressive malignant tumor of the biliary tract system $(1,2)$. In clinical treatment, due to detection difficult at an early stage, most CHOLs are diagnosed

Correspondence to: Dr Yun-Fu Cui, Department of Hepatopancreatobiliary Surgery, Second Affiliated Hospital of Harbin Medical University, Harbin, Heilongjiang 150086, P.R. China E-mail: yfcui777@hotmail.com

*Contributed equally

Key words: competing endogenous RNAs, survival-associate, network, cholangiocarcinoma at an advanced stage, which leads to high mortality, shorter survival and poor prognosis $(3,4)$. Therefore, identifying potential molecular biomarkers is beneficial for CHOL diagnosis, patient prognosis and targeting treatments $(5,6)$, and can promote understanding of the pathological mechanism of CHOL. Long non-coding RNAs (lncRNAs), as transcripts with more than 200 nucleotides in length, play key roles in transcriptionally and post-transcriptionally regulating gene expression (7-9). RNAs have been found involved in multiple cancers by influencing a wide range of functions, such as cell proliferation, cell apoptosis and cell invasion (10-13). Recent studies reported that lncRNAs may be potential diagnostic and prognostic biomarkers for CHOL patients $(14,15)$. Though the roles of lncRNAs acting as biomarkers have been detected, the potential mechanisms are not fully understood in CHOL.

Recent studies revealed that IncRNAs with microRNA (miRNA) responsive elements (MRE) can act as competing endogenous RNAs (ceRNAs) to compete with mRNAs for binding miRNAs, and thus affect the expression level of genes (16-18). The abnormal regulation of ceRNAs relate to many cancers, such as breast, colorectal, gastric and lung cancer (19-23). For example, CXCR as a ceRNA promotes metastasis, proliferation and survival of MCF-7 cells by controlling miRNA activities (24). ROR-Nanog can function as a ceRNA pair and compete for miR-145 predicting poor clinical outcome of pancreatic cancer patients (25). To detect the competing roles of ceRNAs in disease pathology processes, Salmena et al focused on constructing a large-scale ceRNA regulatory network (26). Broad ceRNA interaction network was also identified by considering the ceRNA pairs who significantly shared common miRNAs, and revealed the switch roles of ceRNAs between breast cancer and normal tissues (27). Li et al also demonstrated that the dysregulated interactions in ceRNA network are responsible for high-risk cancers (28).

In the present study, to identify the potential ceRNA interactions contributing to patient survival, we constructed a global ceRNA regulatory network that dysregulated between CHOL and normal tissues. A total of 7 key ceRNA clusters were identified. The Cluster23 involved cell cycle, cell 


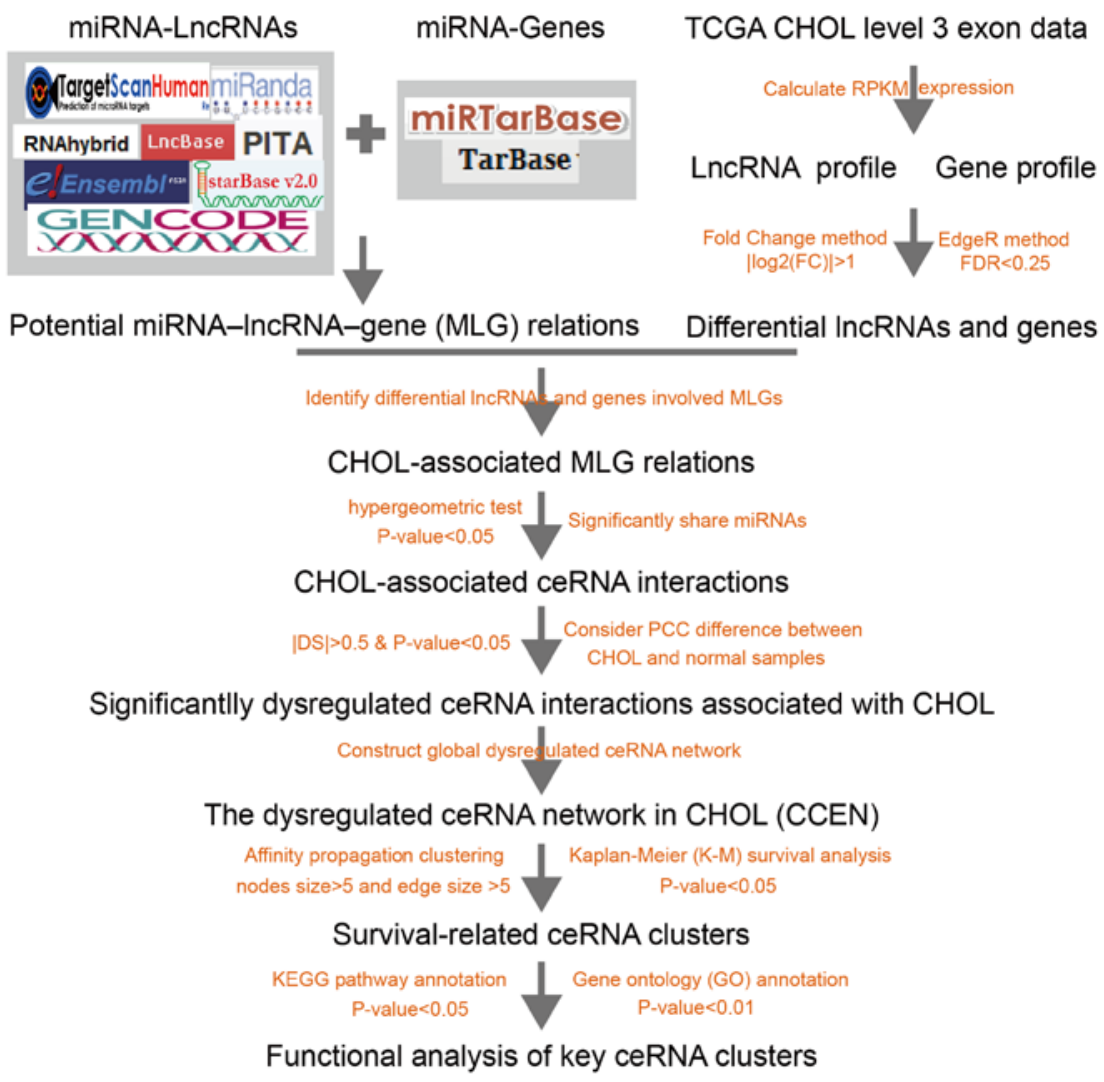

Figure 1. The work schedule.

division, cell proliferation and cell death processes, and mediated the regulation of 'Pathway in cancer'. Another key cluster: Cluster32, was found involved in cell proliferation, apoptosis and programmed cell death processes, and associated with Neurotrophin signaling, focal adhesion and MAPK signaling pathway. This study provides further insights into understanding the competitive mechanism of ceRNAs in affecting the survival of CHOL patients, and may has important clinical significance for screening diagnostic markers.

\section{Materials and methods}

\section{Materials}

LncRNA and miRNA sequences. We obtained the gene annotation of lncRNAs from GENCODE v7 GTF file (29) and the sequences of lncRNAs from Ensembl database (GRCH37) (30). A total of 9,532 lncRNAs with length $>200$ nt were considered. The sequences of mature miRNAs were identified from miRBase (31) and 2,857 miRNAs were finally considered.

Verification of miRNA-target interactions. We obtained the experimentally verified miRNA-gene interactions from mirTarBase (V4.5) (32) and TarBase (V6.0) (33). A total of 3,566 miRNA-gene relation pairs were identified. The experimentally supported miRNA-lncRNA interactions were identified from DIANA-LncBase (34) and starBas (V2.0) (35).

Differential genes and lncRNAs. We downloaded the RNA-seq V2 datasets of cholangiocarcinoma (CHOL), with quantile-normalized and background-corrected at level three, from The Cancer Genome Atlas (TCGA) database (http://tcgadata.nci.nih.gov/). The reads per kilobases per million read
(RPKM) values of genes or lncRNAs can be calculated from exon read count data, the formula is as follows:

$$
R P K M=\frac{A C \times 10^{9}}{S C \times L}
$$

In the above formula, $\mathrm{AC}$ represented the count of reads that mapped into all exons of an lncRNA (gene), and SC was the count of reads that mapped into all exons of a sample. The false discovery rate (FDR) of edgeR method and the ratio of fold change (FC) method were used to level differential expression. One gene or IncRNA was considered to be differentially expressed with FDR $<0.25$ and $\mid \log 2 \mathrm{FCl}>1$. A total of 4,912 genes and 193 lncRNAs were differentially expressed.

\section{Methods}

Identification of potential miRNA-lncRNA-gene relations. There are four steps to identify potential ceRNA interactions: i) we predicted miRNA-lncRNA interactions based on sequence matching algorithms, such as miRanda (36), RNAhybrid (V2.1.1) (37),PITA (38) and TargetScan (V6.0) (39), ii) to extract functional interactions, we analyzed if lncRNA sequences had the binding sites of miRNAs, by integrating the genome coordinates of CLIP-seq peaks (40), then 39,762 miRNA-lncRNA interactions were obtained, iii) by using 3,566 miRNA-gene interactions and 39,762 miRNA-lncRNA interactions, we identified 526,173 potential miRNA-lncRNA-gene (MLG) relations.

Identification of phenotype-associated ceRNA interactions. We obtained the phenotype-associated MLG relations 
Table I. The clusters associated with survival.

\begin{tabular}{|c|c|c|c|}
\hline Cluster ID & LncRNA & Gene & $\begin{array}{l}\text { Log-rank } \\
\text { (P-value) }\end{array}$ \\
\hline cluster23 & $\begin{array}{l}\text { RP4-564F22.2, HCG18, LOC678655, RP11-436I9.2, } \\
\text { CTD-2561B21.9, C12orf47, CTC-228N24.3, } \\
\text { AC092296.1, RP11-403I13.5, H19, MEG3 }\end{array}$ & $\begin{array}{l}\text { UBE2I, IL8, HMGN1, PLP2, FSCN1, DAB2IP, } \\
\text { CCNE1, FAR1, CDK19, PKM, MEIS2, C11 orf9, } \\
\text { HRSP12, CD44, PGF, BCL2, TGFB2 }\end{array}$ & 4.63E-05 \\
\hline cluster24 & $\begin{array}{l}\text { LOC678655, H19, RP11-622K12.1, AC092296.1, } \\
\text { C12orf47, RP11-875O11.1, AC108488.3, } \\
\text { RP11-403I13.5, RP11-444D3.1, MEG3 }\end{array}$ & $\begin{array}{l}\text { RAC1, RHOC, MYLIP, UBE2S, PLAT, JAG1, } \\
\text { MAP3K10, SULF1, FZD7, E2F5, SOX9, SOX4, } \\
\text { ACVR1, CYP1A1 }\end{array}$ & 4.63E-05 \\
\hline cluster42 & $\begin{array}{l}\text { HCG18, MEG3, H19, TUG1, RP11-2C24.4, } \\
\text { LINC00152, C12orf47, RP11-444D3.1, AC092296.1, } \\
\text { ZNFX1-AS1, RP11-622K12.1, PVT1, CTC-504A5.1, } \\
\text { CTD-2561B21.9, RP4-564F22.2, RP11-43619.2 }\end{array}$ & $\begin{array}{l}\text { LIF, CHD4, UBE2I, C2orf18, CYP7A1, CCND3, } \\
\text { ADAM17, HNF4A, POGK, PLEC, GALNT7, } \\
\text { CCNE1, SRGAP1, ARID4B, ARL2, CDH1, } \\
\text { RHOC, COL1A2, PEA15, TPM1, PDLIM7 }\end{array}$ & 4.63E-05 \\
\hline cluster44 & $\begin{array}{l}\text { RP11-300J18.3, RP11-436I9.2, RP4-781K5.7, } \\
\text { SLC38A3, RP11-91K9.1, RP11-403I13.5, } \\
\text { CTC-504A5.1, H19 }\end{array}$ & $\begin{array}{l}\text { GALNT10, ESR1, HCN2, AURKB, CYP24A1, } \\
\text { PRC1, HADH, PARP8, GDAP1 }\end{array}$ & 4.63E-05 \\
\hline cluster43 & $\begin{array}{l}\text { LOC678655, LINC00152, C12orf47, RP11-2C24.4, } \\
\text { AC092296.1, ZNFX1-AS1, CTC-504A5.1, } \\
\text { RP11-622K12.1, RP4-564F22.2, RP11-444D3.1, } \\
\text { TUG1, SNHG6, PVT1 }\end{array}$ & $\begin{array}{l}\text { MFSD10, MYH9, MRE11A, ZNF384, SLC12A2, } \\
\text { COL4A1, SKAP2, TCEAL1, EGLN3, MTA2, } \\
\text { ARID4B, TPM2, RASA1, FGFR3, PLAT, } \\
\text { IGF2BP1, SLC7A1, ABCG2, CCND3, S100B }\end{array}$ & 0.00236 \\
\hline cluster32 & $\begin{array}{l}\text { RP11-622K12.1, MEG3, RP11-2C24.4, RP4-564F22.2, } \\
\text { C12orf47, PVT1, H19, AC108488.3, RP11-436I9.2, } \\
\text { LOC678655, HCG18, RP11-91K9.1, RP11-279F6.1, } \\
\text { AC092296.1, RP11-444D3.1 }\end{array}$ & $\begin{array}{l}\text { BCL2, RAC1, DSTYK, CDCP1, SETD4, TP53, } \\
\text { HBXIP, HNRPDL, SOX9, MUC1, ARID4B, } \\
\text { TMED3, SOX4, CHEK1, SPARC, STMN1, } \\
\text { PEA15, DMD, TNC, IL8 }\end{array}$ & 0.0117 \\
\hline cluster 40 & $\begin{array}{l}\text { AC108488.3, HCG18, RP11-875O11.1, LINC00152, } \\
\text { TUG1, LOC678655, PVT1, CTD-2561B21.9, } \\
\text { RP11-444D3.1, C12orf47, AC092296.1, } \\
\text { RP11-436I9.2, RP11-2C24.4, ALDH1L1-AS2, } \\
\text { H19, MEG3, RP11-91K9.1 }\end{array}$ & $\begin{array}{l}\text { ARHGEF3, PPARA, ANXA11, MMP15, ALDOA, } \\
\text { KRT7, DMTF1, PKMYT1, ISYNA1, ANKIB1, } \\
\text { AP1M2, MYO10, JAG1, EHD2, THRA, } \\
\text { PEA15, GALNT7, RARG, TSPAN15, BCL2, } \\
\text { CCNE1, SULF1, DVL2, SOD1, CCND2, } \\
\text { PDLIM7, TMEM109, COL15A1, PTPN13, CFL2 }\end{array}$ & 0.0251 \\
\hline
\end{tabular}

that involved 4,912 differential genes and 193 differential lncRNAs from 526,173 potential MLG relations. Then hypergeometric method was used to test the enrichment significance of miRNAs between one differential gene and one differential lncRNA. One ceRNA pair was considered as a candidate with $\mathrm{P}_{\mathrm{G}, \mathrm{L}}<0.05$. The enrichment significance can be calculated by using the following formula:

$$
P_{G, L}=1-\sum_{j=0}^{k-1} \frac{\left(\begin{array}{c}
i \\
j
\end{array}\right)\left(\begin{array}{c}
m-i \\
n-j
\end{array}\right)}{\left(\begin{array}{c}
m \\
n
\end{array}\right)}
$$

In the above formula, $m$ represents the total number of mature miRNAs in miRBase, $\mathrm{n}$ is the number of miRNAs targeting gene $G, i$ is the number of miRNAs targeting lncRNA $L, k$ is the number of miRNAs shared by $G$ and $L$. We identified 932 candidate ceRNA pairs that associated with the phenotype, containing 427 genes and 34 lncRNAs.

Evaluation of significantly dysregulated ceRNA interactions. We considered one ceRNA interaction was dysregulated by estimating the difference score $(D S)$ of Pearson's correlation coefficients $(P C C)$ between cancer samples and normal samples. The formula is as follows:
$D S_{G, L}=P C C_{\text {cancer }}(G, L)-P C C_{\text {normal }}(G, L)$

The $\mathrm{PCC}_{\text {cancer }}(G, L)$ and $\mathrm{PCC}_{\text {normal }}(G, L)$ was the $P C C$ between gene $G$ and lncRNA $L$ in cancer samples and normal samples, respectively. The $D S_{G, L}$ was the differential score of $P C C$ between gene $G$ and lncRNA $L$. The higher $D S_{G, L}$ and lower $D S_{G, L}$, respectively, indicated increasing positive and negative interaction in cancer samples. We called it 'gain interaction' when $D S_{G, L}>0.5$ and 'loss interaction' when $D S_{G, L}<-0.5$.

To evaluate the statistical significance of $D S_{G, L}$, we randomly disturbed the normal and cancer labels of all samples while keeping the sample size of normal and cancer unchanged. We performed this process 1,000 times and re-estimated the random $D S_{G, L}$ scores. The statistical significance can be formulated as follows:

P-value $=N\left(\right.$ random $D S_{G, L}>$ true $\left.D S_{G, L}\right) / 1,000$

One ceRNA pair was significantly dysregulated with P-value <0.05. We identified 504 ceRNA pairs with 33 lncRNAs and 298 genes, which significantly dysregulated between two different phenotypes. Finally, 107 ceRNA pairs 


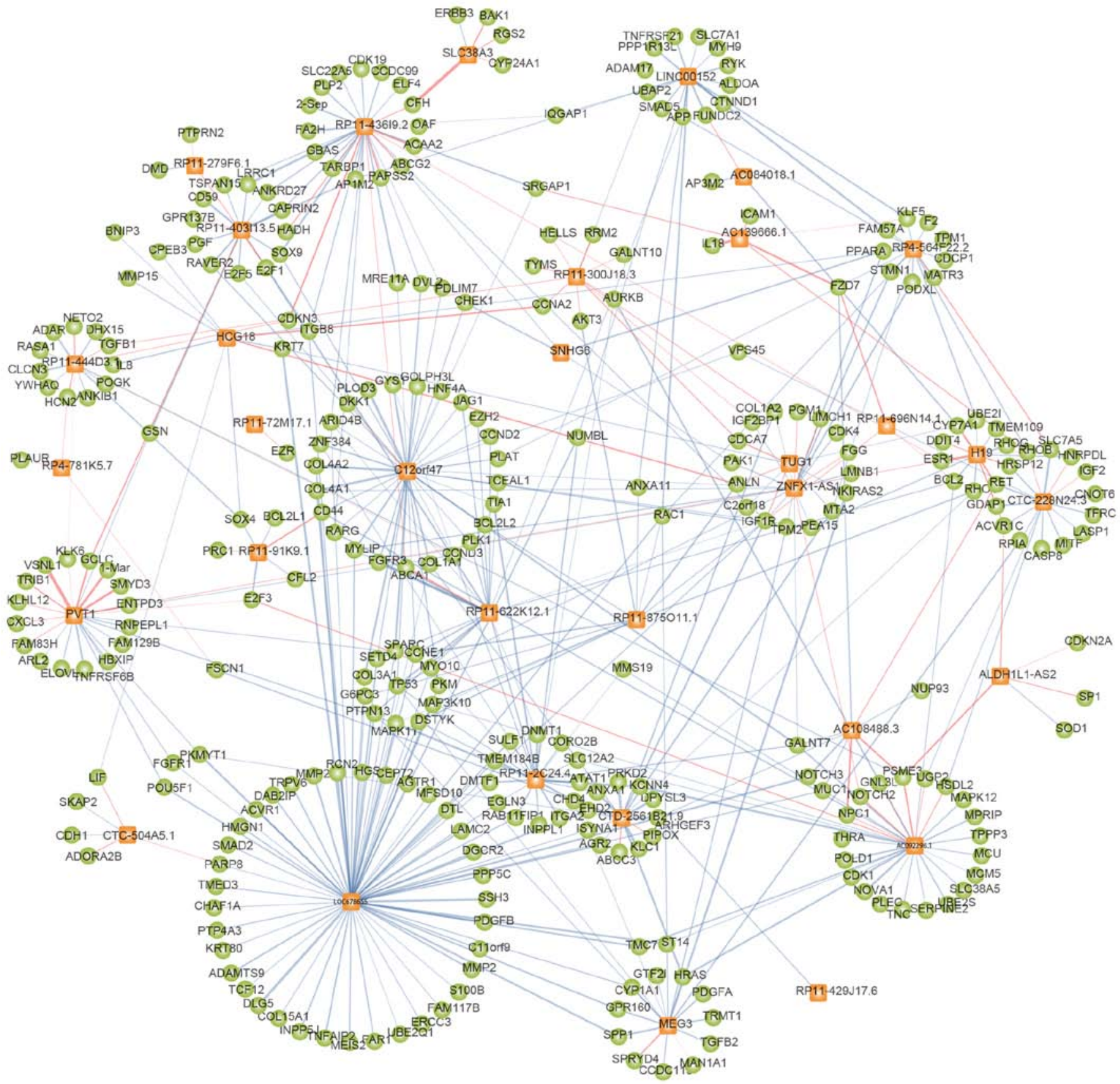

Figure 2. The dysregulated ceRNA network in CHOL (CCEN). The green (circle) and orange (square) nodes represent genes and lncRNAs, respectively. The blue and red lines represent 'loss' and 'gain' interactions, respectively. The width of line is proportional to the DS score of ceRNA.

with gain interaction and 396 ceRNA pairs with loss interactions were obtained.

Cluster functional ceRNA modules. We used an R package 'apcluster' (41) to cluster functional ceRNA modules. The 'negDistMat' function firstly created a squared negative distance matrix with $D S_{G, L}$ scores of 504 ceRNA pairs as parameters. The 'apcluster' function was secondly used, with distance matrix as input, to perform affinity propagation clustering process. This method produced clusters by iteratively maximizing their similarities. We obtained a total of 48 ceRNA clusters and only considered 41 clusters with nodes size $>5$.

Survival analysis. We performed Kaplan-Meier (K-M) method to estimate cumulative survival rates and log-rank test to evaluate the difference in overall survival between different group patients. A P-value of $<0.05$ was considered to indicate statistical significance.

\section{Results}

The construction of CCEN. The dysregulated ceRNA network in CHOL (CCEN) was constructed, based on lncRNA-gene interactions with significant $D S_{G, L}$ scores. This network contained 294 genes, 33 lncRNAs and 504 edges (Fig. 1). The gain and loss interaction modes of lncRNAs and genes can be shown in CCEN, which characterized that two nodes linked to common edge had increasing positive and negative mode in CHOL, respectively. A total of 108 'gain interaction' and 396 'loss interaction' were obtained (data not shown). 
A
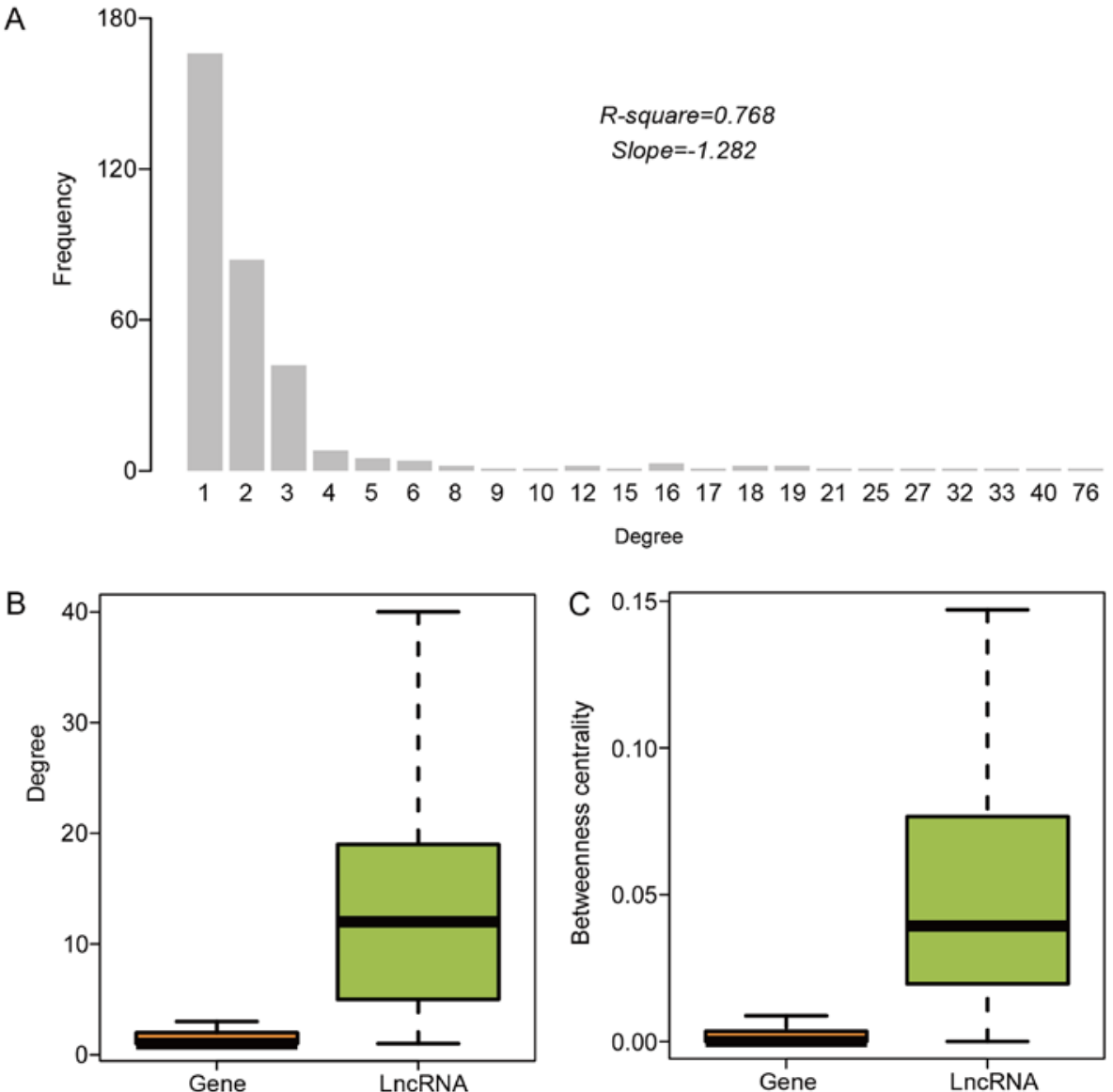

Figure 3. Analysis of CCEN network properties. (A) The degree distribution of CCEN nodes. (B) The degrees of lncRNAs and genes in CCEN. (C) The betweenness centrality of IncRNAs and genes in CCEN.

Survival-related ceRNA clusters. The 'Affinity Propagation' method was used to cluster functional ceRNA modules from CCEN. We obtained 49 clusters and only considered 32 clusters with node size $>5$ and edge size $>5$. By performing 'K-M' survival analysis, 7 key clusters can significantly distinguish survival rates between two different groups (Table I).

Function analysis of key ceRNA clusters. We performed GO and pathway functional annotation for each key ceRNA cluster, by using functional annotation tool of DAVID (42). Genes within one cluster were used. The annotation results are shown (data not shown).

\section{Discussion}

LncRNAs have been shown to play key roles in regulating gene expression by competing for miRNAs, and the ceRNA relations formed can contribute to the development and progression of multiple cancers $(17,43)$. To explore the competitive relationship of $\operatorname{lncRNAs}$ and genes, we firstly established the potential miRNA-lncRNA-gene set. The experimentally verified miRNA-mRNA interactions werw collected from mirTarBase (V4.5) and TarBase (V6.0). However, detecting the interactions between miRNAs and lncRNAs is a great challenge. Several studies identified physical miRNA-lncRNA interactions by evaluating the match extent and conserved seed regions $(44,45)$. The functional miRNA-lncRNA inter- actions were predicted by identifying the SNPs that affected the binding of miRNAs and IncRNAs (46). In addition, the experimentally verified and predicted miRNA-lncRNA interactions were recently identified in DIANA-LncBase and starBase (V2.0). Here, we applied the traditional miRNA target prediction methods by analyzing AGO-CLIP-seq data set and integrated the verified interactions to enhance the functional reliabilities of candidate miRNA-lncRNA interactions. The potential ceRNAs data set was finally obtained by integrating miRNA-mRNA and miRNA-lncRNA interactions. Because lncRNAs can compete with endogenous mRNAs for binding miRNAs, we further used hypergeometric method to identify the high-confidence ceRNAs.

We constructed a bilayer network named CCEN and dissected the characteristics of CCEN to reveal the potential competitive roles of ceRNAs. The CCEN conformed to powerlaw distribution with $\mathrm{R}^{2}=0.768$ and slope $=-1.282$ (Fig. 2). The average degree of genes in network was 1.69 , whereas that of lncRNAs was up to 15.27 (Fig. 2). The average betweenness centrality of genes was 0.0039 , whereas that of lncRNAs (0.0598) was about 15-fold higher than the genes (Fig. 2). The IncRNA PVT1 with a degree of 25 and betweenness centrality of 0.1119 were higher than each average level, have been demonstrated to be associated with many cancers. For example, PVT1 can frequently amplify or mutate to promote the pathophysiological process of ovarian, breast cancer, Burkitt's lymphomas and murine plasmacytomas (47-49). 
A

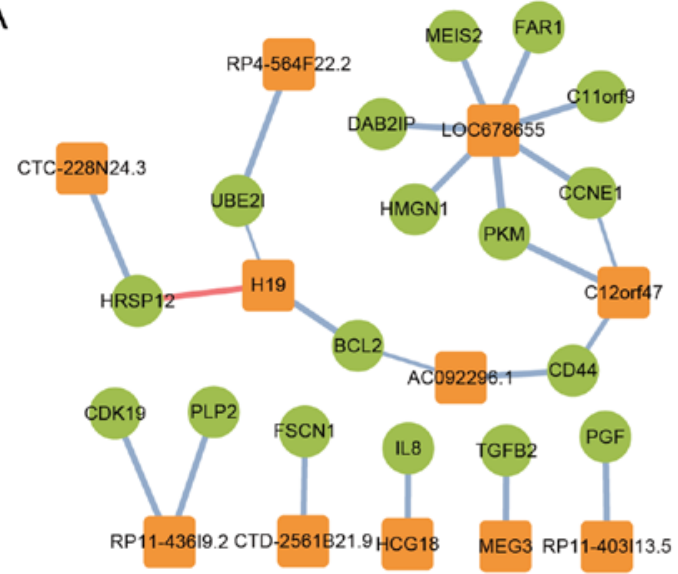

B

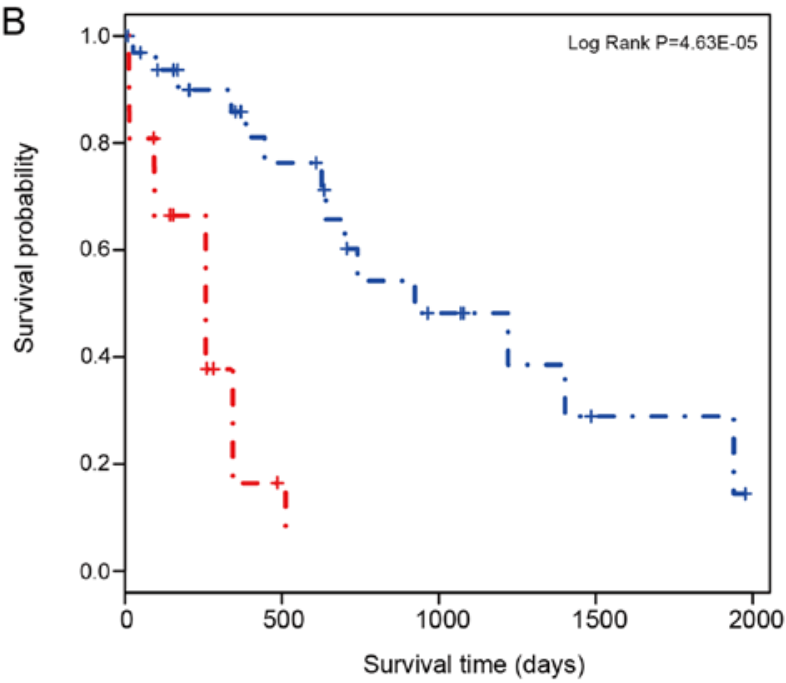

C

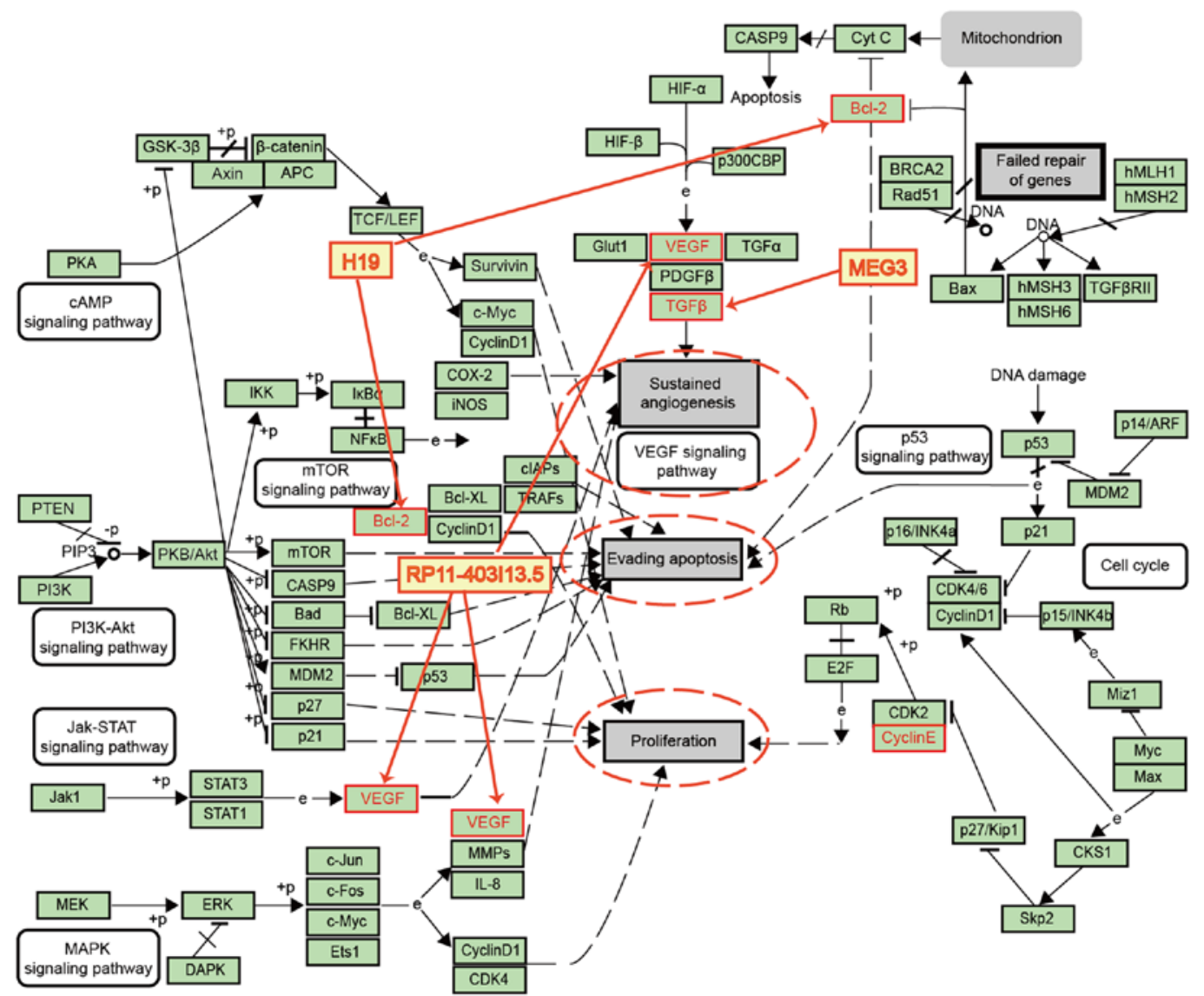

Figure 4. Analysis of survival-associated Cluster23. (A) The network structure of module cluster23. (B) 'K-M' survival analysis of cluster23 members. (C) The annotated pathway 'Pathway in cancer'.

The IncRNA ZNFX1-AS1 with degree of 21 and betweenness centrality of 0.0766 , is a putative tumor suppressor downregulated in breast cancer and ductal carcinoma (50). It suggested that lncRNAs tended to compete with multiple genes and played crucial bridge roles for conveying information in cancers

To obtain functional ceRNA modules that associated with patient survival, we applied affinity propagation cluster method to CCEN and identified 48 functional clusters. As the few nodes and edges in a single cluster were difficult to connect in function, we retained 32 clusters with both node size $>5$ and edge size $>5$. Then K-M survival analysis method was used, to estimate if the elements in one cluster can distinguish the survival rates between two different groups. The Cluster23 had 11 lncRNAs and 17 genes (Fig. 3A), which was one of the most significant clusters related to $\mathrm{CHOL}$ patient survival (P-value $=4.63 \mathrm{E}-05$, Fig. 3B). We extracted the subnetwork consisted of Cluster 23 members in CCEN, most ceRNA pairs shared one or more competitors with others, and only 4 ceRNA pairs were specific in competition. In GO functional analysis (data not shown), Cluster23 was associated with many important biological processes, such as cell cycle, 

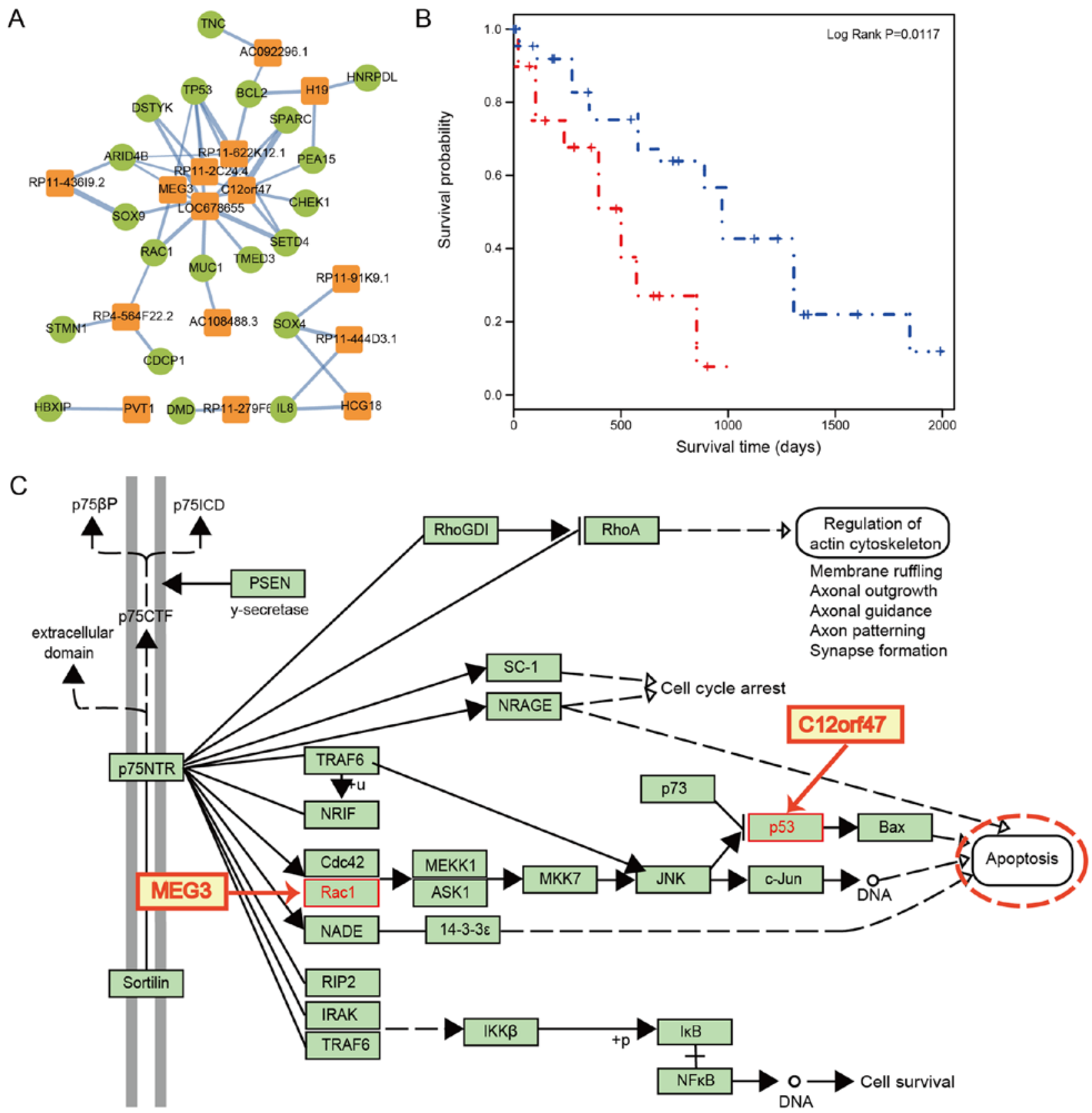

Figure 5. Analysis of survival-associated Cluster32. (A) The network structure of module Cluster32. (B) 'K-M' survival analysis of Cluster32 members. (C) The annotated pathway 'Neurotrophin signaling pathway'.

cell division, cell proliferation, tissue and tube morphogenesis. Interestingly, we also found that all competitive relations except H19-HRSP12 of Cluster23 were 'loss interaction' in CHOL compared to normal tissues. It revealed that the lost of ceRNA competitive relations may promote the development and progression of $\mathrm{CHOL}$, by disturbing the cycle, division or proliferation of cells. To examine the roles of Cluster23 in the pathway, we dissected the most significant pathway 'Pathway in cancer' (P-value $=2.30 \mathrm{E}-04$, data not shown). The Cluster23 members, Bcl-2, TGFB, VEGF and cyclin E, were all located in the central position of pathway and the upstream of other key pathways, such as sustained angiogenesis, proliferation and evading apoptosis process (Fig. 3C). Pugazhenthi et al demonstrated that $\mathrm{H} 19-7$ cells, with Bcl-2 overexpressed, can resist ROS-induced apoptosis (51). A recent study confirmed that MEG3 expression is low in breast tumors with high TGFB2 expression (52). Therefore, H19 and MEG3 negatively regulate Bcl-2 and TGFB2 expression in tumors, respectively, and may contribute to $\mathrm{CHOL}$ phenotype.

Another key survival cluster was Cluster32 (Fig. 4A), with 15 lncRNAs and 20 genes ( $\mathrm{P}=0.012$, Fig. 4B). In this cluster, all competitive relations were 'loss interaction', which may indicate the relationships between dysregulated ceRNA interactions and CHOL phenotype. We found that genes in this cluster involved cell proliferation, apoptosis and programmed cell death processes (data not shown). The result of further pathway enrichment analysis showed that Cluster 32 was significantly associated with Neurotrophin signaling $(\mathrm{P}=0.011)$, focal adhesion $(\mathrm{P}=0.029)$ and MAPK signaling pathway $(\mathrm{P}=0.048)$ (data not shown). These pathways had been confirmed related 
to the development and progression of cancers. For example, the induce of MAPK signaling pathway activity promotes the metastasis of breast cancer cells and enhances cancer malignancy $(53,54)$, inhibiting focal adhesion kinase can induce apoptosis of bladder and breast cancer cells $(55,56)$, the activation of neurotrophin signaling promotes the growth of brain tumor-initiating cells and the metastasis of lung adenocarcinoma $(57,58)$. Then we dissected the regulatory mechanism of ceRNA interactions in CHOL through analyzing Neurotrophin signaling pathway (Fig. 5). In this pathway, gene Rac1 was located in the initial position, lncRNA MEG3 targeting Rac1 was demonstrated to suppress migration and invasion of thyroid carcinoma (59). TP53 was a downstream gene of Rac1, the abnormal expression of which contributes to many cancers, such as large B-cell lymphoma (60), gastrointestinal cancer (61), and lymphocytic leukemia (62). The lncRNA C12orf47 competed with TP53 and influenced its expression, which may mediate the apoptosis of CHOL by disturbing the Neurotrophin signaling pathway. These results suggested that the Cluster32 we identified may control the survival of CHOL patients, by mediating ceRNA regulation in cell functions.

In conclusion, although the results of the present study require further experimental verification, the results provide further insights into understanding the loss of ceRNA interactions in affecting the survival of CHOL patients.

\section{References}

1. Torok N and Gores GJ: Cholangiocarcinoma. Semin Gastrointest Dis 12: 125-132, 2001.

2. Vauthey JN and Blumgart LH: Recent advances in the management of cholangiocarcinomas. Semin Liver Dis 14 109-114, 1994.

3. Rizvi S and Gores GJ: Pathogenesis, diagnosis, and management of cholangiocarcinoma. Gastroenterology 145: 1215-1229, 2013.

4. Singh P and Patel T: Advances in the diagnosis, evaluation and management of cholangiocarcinoma. Curr Opin Gastroenterol 22: 294-299, 2006.

5. Welzel TM, Graubard BI, Zeuzem S, El-Serag HB, Davila JA and McGlynn KA: Metabolic syndrome increases the risk of primary liver cancer in the United States: A study in the SEERMedicare database. Hepatology 54: 463-471, 2011.

6. Seeree P, Pearngam P, Kumkate $S$ and Janvilisri T: An omics perspective on molecular biomarkers for diagnosis, prognosis, and therapeutics of cholangiocarcinoma. Int J Genomics 2015 179528, 2015.

7. Mercer TR, Dinger ME and Mattick JS: Long non-coding RNAs: Insights into functions. Nat Rev Genet 10: 155-159, 2009

8. Hawkins PG and Morris KV: Transcriptional regulation of Oct4 by a long non-coding RNA antisense to Oct4-pseudogene 5 . Transcription 1: 165-175, 2010

9. Tripathi V, Ellis JD, Shen Z, Song DY, Pan Q, Watt AT, Freier SM Bennett CF, Sharma A, Bubulya PA, et al: The nuclear-retained noncoding RNA MALAT1 regulates alternative splicing by modulating SR splicing factor phosphorylation. Mol Cell 39: 925-938, 2010.

10. Huarte M: The emerging role of lncRNAs in cancer. Nat Med 21: 1253-1261, 2015.

11. Gibb EA, Brown CJ and Lam WL: The functional role of long non-coding RNA in human carcinomas. Mol Cancer 10: 38 , 2011.

12. Gutschner T and Diederichs S: The hallmarks of cancer: A long non-coding RNA point of view. RNA Biol 9: 703-719, 2012.

13. Zhang H, Chen Z, Wang X, Huang Z, He Z and Chen Y: Long non-coding RNA: A new player in cancer. J Hematol Oncol 6 : 37, 2013.

14. Ma SL, Li AJ, Hu ZY, Shang FS and Wu MC: Co-expression of the carbamoyl-phosphate synthase 1 gene and its long non-coding RNA correlates with poor prognosis of patients with intrahepatic cholangiocarcinoma. Mol Med Rep 12: 7915-7926, 2015.
15. Wang J, Xie H, Ling Q, Lu D, Lv Z, Zhuang R, Liu Z, Wei X, Zhou L, Xu X, et al: Coding-noncoding gene expression in intrahepatic cholangiocarcinoma. Transl Res 168: 107-121, 2016.

16. Tay Y, Kats L, Salmena L, Weiss D, Tan SM, Ala U, Karreth F, Poliseno L, Provero P, Di Cunto F, et al: Coding-independent regulation of the tumor suppressor PTEN by competing endogenous mRNAs. Cell 147: 344-357, 2011.

17. Cesana M, Cacchiarelli D, Legnini I, Santini T, Sthandier O, Chinappi M, Tramontano A and Bozzoni I: A long noncoding RNA controls muscle differentiation by functioning as a competing endogenous RNA. Cell 147: 358-369, 2011.

18. Sumazin P, Yang X, Chiu HS, Chung WJ, Iyer A, Llobet-Navas D, Rajbhandari P, Bansal M, Guarnieri P, Silva J, et al: An extensive microRNA-mediated network of RNA-RNA interactions regulates established oncogenic pathways in glioblastoma. Cell 147: 370-381, 2011

19. Tay Y, Karreth FA and Pandolfi PP: Aberrant ceRNA activity drives lung cancer. Cell Res 24: 259-260, 2014.

20. Hu Y, Tian H, Xu J and Fang JY: Roles of competing endogenous RNAs in gastric cancer. Brief Funct Genomics: Sep 24, 2015 (Epub ahead of print).

21. Xia T, Liao Q, Jiang X, Shao Y, Xiao B, Xi Y and Guo J: Long noncoding RNA associated-competing endogenous RNAs in gastric cancer. Sci Rep 4: 6088, 2014.

22. Liang WC, Fu WM, Wong CW, Wang Y, Wang WM, Hu GX, Zhang L, Xiao LJ, Wan DC, Zhang JF, et al: The lncRNA H19 promotes epithelial to mesenchymal transition by functioning as miRNA sponges in colorectal cancer. Oncotarget 6: 22513-22525, 2015.

23. Yang J, Li T, Gao C, Lv X, Liu K, Song H, Xing Y and Xi T: FOXO1 3'UTR functions as a ceRNA in repressing the metastases of breast cancer cells via regulating miRNA activity. FEBS Lett 588: 3218-3224, 2014.

24. Zheng T, Chou J, Zhang F, Liu Y, Ni H, Li X, Zheng L, Tang T, Jin L and Xi T: CXCR4 3'UTR functions as a ceRNA in promoting metastasis, proliferation and survival of MCF-7 cells by regulating miR-146a activity. Eur J Cell Biol 94: 458-469, 2015.

25. Gao S, Wang P, Hua Y, Xi H, Meng Z, Liu T, Chen Z and Liu L: ROR functions as a ceRNA to regulate Nanog expression by sponging miR-145 and predicts poor prognosis in pancreatic cancer. Oncotarget 7: 1608-1618, 2016.

26. Salmena L, Poliseno L, Tay Y, Kats L and Pandolfi PP: A ceRNA hypothesis: The Rosetta Stone of a hidden RNA language? Cell 146: 353-358, 2011.

27. Paci P, Colombo T and Farina L: Computational analysis identifies a sponge interaction network between long non-coding RNAs and messenger RNAs in human breast cancer. BMC Syst Biol 8: 83, 2014.

28. Li L, Wang D, Xue M, Mi X, Liang Y and Wang P: 3'UTR shortening identifies high-risk cancers with targeted dysregulation of the ceRNA network. Sci Rep 4: 5406, 2014.

29. Derrien T, Johnson R, Bussotti G, Tanzer A, Djebali S, Tilgner H, Guernec G, Martin D, Merkel A, Knowles DG, et al: The GENCODE v7 catalog of human long noncoding RNAs: Analysis of their gene structure, evolution, and expression. Genome Res 22: 1775-1789, 2012.

30. Kersey PJ, Lawson D, Birney E, Derwent PS, Haimel M, Herrero J, Keenan S, Kerhornou A, Koscielny G, Kähäri A, et al: Ensembl Genomes: Extending Ensembl across the taxonomic space. Nucleic Acids Res 38: D563-D569, 2010.

31. Kozomara A and Griffiths-Jones S: miRBase: Integrating microRNA annotation and deep-sequencing data. Nucleic Acids Res 39: D152-D157, 2011.

32. Hsu SD, Tseng YT, Shrestha S, Lin YL, Khaleel A, Chou CH, Chu CF, Huang HY, Lin CM, Ho SY, et al: miRTarBase update 2014: An information resource for experimentally validated miRNA-target interactions. Nucleic Acids Res 42: D78-D85, 2014.

33. Vergoulis T, Vlachos IS, Alexiou P, Georgakilas G, Maragkakis M, Reczko M, Gerangelos S, Koziris N, Dalamagas T and Hatzigeorgiou AG: TarBase 6.0: Capturing the exponential growth of miRNA targets with experimental support. Nucleic Acids Res 40: D222-D229, 2012.

34. Paraskevopoulou MD, Georgakilas G, Kostoulas N, Reczko M, Maragkakis M, Dalamagas TM and Hatzigeorgiou AG: DIANA-LncBase: Experimentally verified and computationally predicted microRNA targets on long non-coding RNAs. Nucleic Acids Res 41: D239-D245, 2013. 
35. Yang JH, Li JH, Shao P, Zhou H, Chen YQ and Qu LH: starBase: A database for exploring microRNA-mRNA interaction maps from Argonaute CLIP-Seq and Degradome-Seq data. Nucleic Acids Res 39: D202-D209, 2011.

36. Betel D, Wilson M, Gabow A, Marks DS and Sander C: The microRNA.org resource: Targets and expression. Nucleic Acids Res 36: D149-D153, 2008.

37. Rehmsmeier M, Steffen P, Hochsmann M and Giegerich R: Fast and effective prediction of microRNA/target duplexes. RNA 10 $1507-1517,2004$

38. Kertesz M, Iovino N, Unnerstall U, Gaul U and Segal E: The role of site accessibility in microRNA target recognition. Nat Genet 39: 1278-1284, 2007.

39. Friedman RC, Farh KK, Burge CB and Bartel DP: Most mammalian mRNAs are conserved targets of microRNAs. Genome Res 19: 92-105, 2009.

40. Hafner M, Landthaler M, Burger L, Khorshid M, Hausser J, Berninger P, Rothballer A, Ascano M Jr, Jungkamp AC, Munschauer M, et al: Transcriptome-wide identification of RNA-binding protein and microRNA target sites by PAR-CLIP Cell 141: 129-141, 2010.

41. Bodenhofer U, Kothmeier A and Hochreiter S: APCluster: An $\mathrm{R}$ package for affinity propagation clustering. Bioinformatics 27 2463-2464, 2011.

42. Huang DW, Sherman BT, Tan Q, Collins JR, Alvord WG, Roayaei J, Stephens R, Baseler MW, Lane HC and Lempicki RA: The DAVID Gene Functional Classification Tool: A novel biological module-centric algorithm to functionally analyze large gene lists. Genome Biol 8: R183, 2007.

43. Wang Y, Xu Z, Jiang J, Xu C, Kang J, Xiao L, Wu M, Xiong J, Guo $X$ and Liu H: Endogenous miRNA sponge lincRNA-RoR regulates Oct4, Nanog, and Sox 2 in human embryonic stem cel self-renewal. Dev Cell 25: 69-80, 2013.

44. Jeggari A, Marks DS and Larsson E: miRcode: A map of putative microRNA target sites in the long non-coding transcriptome. Bioinformatics 28: 2062-2063, 2012.

45. Jalali S, Bhartiya D, Lalwani MK, Sivasubbu S and Scaria V: Systematic transcriptome wide analysis of lncRNA-miRNA interactions. PLoS One 8: e53823, 2013

46. Gong J, Liu W, Zhang J, Miao X and Guo AY: lncRNASNP: A database of SNPs in lncRNAs and their potential functions in human and mouse. Nucleic Acids Res 43: D181-D186, 2015.

47. Guan Y, Kuo WL, Stilwell JL, Takano H, Lapuk AV, Fridlyand J, Mao JH, Yu M, Miller MA, Santos JL, et al: Amplification of PVT1 contributes to the pathophysiology of ovarian and breast cancer. Clin Cancer Res 13: 5745-5755, 2007.

48. Carramusa L, Contino F, Ferro A, Minafra L, Perconti G, Giallongo A and Feo S: The PVT-1 oncogene is a Myc protein target that is overexpressed in transformed cells. J Cell Physiol 213: 511-518, 2007.

49. Meyer KB, Maia AT, O'Reilly M, Ghoussaini M,Prathalingam R Porter-Gill P, Ambs S, Prokunina-Olsson L, Carroll J and Ponder BA: A functional variant at a prostate cancer predisposition locus at $8 \mathrm{q} 24$ is associated with PVT1 expression. PLoS Genet 7: e1002165, 2011
50. Askarian-Amiri ME, Crawford J, French JD, Smart CE, Smith MA, Clark MB, Ru K, Mercer TR, Thompson ER, Lakhani SR, et al: SNORD-host RNA Zfas1 is a regulator of mammary development and a potential marker for breast cancer. RNA 17: 878-891, 2011.

51. Pugazhenthi S, Nesterova A, Jambal P, Audesirk G, Kern M, Cabell L, Eves E, Rosner MR, Boxer LM and Reusch JE: Oxidative stress-mediated down-regulation of bcl-2 promoter in hippocampal neurons. J Neurochem 84: 982-996, 2003.

52. Mondal T, Subhash S, Vaid R, Enroth S, Uday S, Reinius B, Mitra S, Mohammed A, James AR, Hoberg E, et al: MEG3 long noncoding RNA regulates the TGF- $\beta$ pathway genes through formation of RNA-DNA triplex structures. Nat Commun 6: 7743, 2015.

53. Chen XY, Zhou J, Luo LP, Han B, Li F, Chen JY, Zhu YF, Chen W and Yu XP: Black rice Anthocyanins suppress metastasis of breast cancer cells by targeting RAS/RAF/MAPK pathway. Biomed Res Int 2015: 414250, 2015.

54. Zhang X, Ma L, Qi J, Shan H, Yu W and Gu Y: MAPK/ERK signaling pathway-induced hyper-O-GlcNAcylation enhances cancer malignancy. Mol Cell Biochem 410: 101-110, 2015.

55. Kong D, Chen F and Sima NI: Inhibition of focal adhesion kinase induces apoptosis in bladder cancer cells via Src and the phosphatidylinositol 3-kinase/Akt pathway. Exp Ther Med 10: 1725-1731, 2015

56. Wu X, Zahari MS, Renuse S, Nirujogi RS, Kim MS, Manda SS, Stearns V, Gabrielson E, Sukumar S and Pandey A: Phosphoproteomic analysis identifies focal adhesion kinase 2 (FAK2) as a potential therapeutic target for tamoxifen resistance in breast cancer. Mol Cell Proteomics 14: 2887-2900, 2015.

57. Lawn S, Krishna N, Pisklakova A, Qu X, Fenstermacher DA, Fournier M, Vrionis FD, Tran N, Chan JA, Kenchappa RS, et al: Neurotrophin signaling via TrkB and TrkC receptors promotes the growth of brain tumor-initiating cells. J Biol Chem 290: 3814-3824, 2015

58. Sinkevicius KW, Kriegel C, Bellaria KJ, Lee J, Lau AN, Leeman KT, Zhou P, Beede AM, Fillmore CM, Caswell D, et al: Neurotrophin receptor TrkB promotes lung adenocarcinoma metastasis. Proc Natl Acad Sci USA 111: 10299-10304, 2014

59. Wang C, Yan G, Zhang Y, Jia X and Bu P: Long non-coding RNA MEG3 suppresses migration and invasion of thyroid carcinoma by targeting of Rac1. Neoplasma 62: 541-549, 2015.

60. Lu TX, Young KH, Xu W and Li JY: TP53 dysfunction in diffuse large B-cell lymphoma. Crit Rev Oncol Hematol 97: 47-55, 2016.

61. Rokavec M, Li H, Jiang L and Hermeking H: The p53/microRNA connection in gastrointestinal cancer. Clin Exp Gastroenterol 7: 395-413, 2014.

62. Malcikova J, Pavlova S, Kozubik KS and Pospisilova S: TP53 mutation analysis in clinical practice: Lessons from chronic lymphocytic leukemia. Hum Mutat 35: 663-671, 2014. 